\title{
Usefulness of magnetic resonance findings of the hypothalamic-pituitary region in the management of short children with growth hormone deficiency: evidence from a longitudinal study
}

\author{
Maria A. Kalina • Barbara Kalina-Faska • \\ Katarzyna Gruszczyńska • Jan Baron • \\ Ewa Malecka-Tendera
}

Received: 9 July 2011 / Accepted: 12 September 2011 / Published online: 21 September 2011

(C) The Author(s) 2011. This article is published with open access at Springerlink.com

\begin{abstract}
Purpose The purpose of this study is to assess the relationship between magnetic resonance images (MRI) of the hypothalamic-pituitary (H-P) region and response to recombinant human growth hormone $(\mathrm{rhGH})$ treatment in short children with growth hormone deficiency, basing on changes of auxologic parameters, as well as to answer the question if MRI may serve for selecting and monitoring the rhGH responders.

Patients and methods The study group comprised 85 children treated with rhGH, aged 7.3-18.7 years, followed for the mean period of 3.2 years (range, 2.1-9.5 years). Auxologic parameters (height deficit hSDS, deviation from the mid-parental height hSDS-mpSDS, bone delay index bone age/chronological age ratio (BA/CA)) were assessed before, during and at the end of rhGH treatment; growth velocity was calculated before and during rhGH therapy. Parameters were correlated with the MRI of the H-P region. Results Structural anomalies of the H-P region were found in $22(25.9 \%)$ children: empty sella syndrome (ESS) in 12 (14.1\%) patients, ectopic posterior pituitary (EPP) in ten
\end{abstract}

M. A. Kalina $(\bowtie) \cdot$ B. Kalina-Faska $\cdot$ E. Małecka-Tendera Department of Paediatrics, Paediatric Endocrinology and Diabetes, Medical University of Silesia,

Upper Silesia Centre for Child's Health ul. Medyków 16, 40-752 Katowice, Poland

e-mail: majak_2001@yahoo.com

K. Gruszczyńska • J. Baron

Department of Radiology and Nuclear Medicine, Medical

University of Silesia,

ul. Medyków 14,

40-752 Katowice, Poland
(11.8\%). Patients' height deficit and their deviation from parental height before rhGH therapy was significantly greater in the EPP group (median hSDS $=-3.8$; hSDS$\operatorname{mpSDS}=-2.5)$, bone age delay was the greatest in the ESS group (median $\mathrm{BA} / \mathrm{CA}=0.69$ ), after therapy - in the EPP group (median $\mathrm{BA} / \mathrm{CA}=0.82$ ). Growth velocity improved in the first year of the rhGH therapy in all groups; however, the most significant acceleration was observed in the EPP group (median delta $\mathrm{hSDS}=0.9$ ), then stabilised and was comparable in all groups.

Conclusions MRI may be helpful in predicting response to the rhGH treatment, providing midline abnormalities are taken into account.

Keywords Growth hormone deficiency · Magnetic resonance $\cdot$ Ectopic posterior pituitary $\cdot$ Empty sella syndrome $\cdot$ Recombinant growth hormone therapy . Children

\section{Introduction}

Treating short children with recombinant human growth hormone (rhGH) is an expensive and long-term modality, hence it has been strived for selecting patients who would benefit the most from such therapy. Minimal criteria for diagnosing growth hormone deficiency (GHD) should include auxologic and biochemical parameters [12]. However, laboratory assays tend to be imperfect due to low reproducibility, intrapersonal and cyclic variability of growth hormone $(\mathrm{GH})$ secretion, hence auxology still remains the most important diagnostic criterion [10, 12$14,23,27]$. It is believed that the greatest benefits of rhGH 
therapy are observed in the youngest children, with significant growth deficiency, slow height velocity and delayed bone age $[1,12]$. It has been suggested that limited propagation of magnetic resonance imaging (MRI) of the hypothalamic-pituitary (H-P) region is one of the reasons for not achieving desirable treatment effects [12]. Magnetic resonance is a choice method for visualising the pituitary region, as it provides high contrast resolution, the possibility of multiplanar imaging, omits artefacts from bone structures and avoids X-ray exposure, being particularly advantageous in children [8]. Usually, MRI is performed as an end-step diagnostic procedure to exclude expansive lesions in the H-P region before initiation of the rhGH treatment. However, it can also be used to visualise congenital structural abnormalities in the central nervous system, which may be associated with growth disturbances. It has been postulated that MRI may be a more accurate indicator of hormonal deficiencies and potential response to the rhGH treatment than laboratory results $[6,9,17,26]$.

Previously, we published that GH secretion did not depend on the size of the pituitary, unless structural defects of the H-P region are taken into account, which was also supported by other reports $[3,15,25]$. The aim of the present study was to assess the relationship between MRI of the H-P region and response to rhGH treatment in GHdeficient children, basing on changes of auxologic parameters during a long-term follow-up, as well as to answer the question if MRI findings may serve for selecting and monitoring the rhGH responders.

\section{Patients and methods}

The studied group comprised 85 children and adolescents (59 boys and 29 girls), ranging from 7.3 to 18.7 years of age at the referral (median age, 14.1 years). The patients were followed in the Department of Paediatrics, Endocrinology and Diabetes of the Medical University of Silesia, Katowice, Poland, for the mean observational period of 3.2 years (range, $2.1-9.5$ years). Inclusion criteria were as follows: short stature (height below 2 standard deviations (SDs) from the population mean), slow height velocity (below tenth centile) and GHD (peak GH concentration below $10 \mathrm{ng} / \mathrm{ml}$ in two stimulation tests). The latter included intravenous administration of insulin in the dosage of $0.1 \mathrm{U} / \mathrm{kg}$ (Actrapid, NovoNordisk) and reaching hypoglycaemic state (serum glucose concentration below $40 \mathrm{mg} / \mathrm{dl}$ ) and after oral administration of clonidine in the dosage of $0.15 \mathrm{mg} / \mathrm{m}^{2}$ body surface (Iporel, Polfa). Concentrations of GH were assayed using chemiluminescent immunometric method (Immulite, Euro/DPC, Llanberis, UK).

Based on inclusion criteria, children were treated with rhGH in the mean dose of $0.03 \mathrm{mg} / \mathrm{kg} / \mathrm{day}$ (Genotropin,
Pfeizer). Appropriate substitution therapy was implemented in the case of deficiency of other pituitary hormones. The rhGH therapy was terminated when slow height velocity was noted (less than $3 \mathrm{~cm}$ per year).

Exclusion criteria comprised chromosomal aberrations, dysmorphic syndromes, bone dysplasia, children born small for gestational age or with intrauterine growth retardation, patients with chronic diseases, acquired growth hormone deficiency, including post-traumatic or post-neoplastic deficiencies.

\section{Anthropometry}

Anthropometric measurements, referred to national growth charts [21], and evaluation of sexual development using Tanner staging were performed every 6 months. The following parameters were derived from anthropometric measurements and assessed before, during and at the end of rhGH treatment: a number of SDs for the height (hSDS), change of hSDS in a year, and the difference of SDs between child's height and the mid-parental height (hSDS-mpSDS).

\section{Imaging procedures}

The MRI of the H-P region was assessed by two radiologists $(\mathrm{KG}, \mathrm{JB})$, unaware of the endocrine diagnosis. The studies were performed using a 1-T scanner (Magnetom Impact, Siemens, Erlangen, Germany), with a head coil, in the coronal and sagittal planes at the level of the pituitary (slice thickness of $3 \mathrm{~mm}$ without gap). The sagittal planes were positioned parallel to the midline on the pilot scan in the coronal plane. SE/T1-weighted sequences were used before and after intravenous administration of the contrast agent (Magnevist, Schering), in the dosage of $0.1 \mathrm{ml} / \mathrm{kg}$ body mass. Parameters of the T1 sequence were as follows: TE, $15 \mathrm{~ms}$; TR, 490-500 ms; FOV, $172 \times$ $230 \mathrm{~mm}$; flip angle (FA), $90^{\circ}$; matrix, $256 \times 256$. No patient required anaesthesia.

Special attention was paid to the visualisation of the pituitary stalk and to the presence of structural anomalies in the H-P area like ectopic posterior pituitary, empty sella syndrome and other midline anomalies. Radiological features of the empty sella syndrome were identified when the sella space was not filled by the pituitary gland completely. Instead, it was occupied at least partially by the cerebrospinal fluid (CSF), with herniation of the suprasellar cisterns below the line of the sella diaphragm (i.e. below the line between the upper margin of the sella tuberculum and the upper part of the sella dorsum). Partial empty sella syndrome was defined when less than half of the sella was filled with CSF, and the pituitary height was more than $2 \mathrm{~mm}$. Total empty sella syndrome was 
diagnosed when more than half of the sella was filled with $\mathrm{CSF}$, and the pituitary height was equal or less than $2 \mathrm{~mm}$ [7].

Other radiological techniques used in the study included wrist and hand X-ray of a non-dominant side in the anteriorposterior projection and the assessment of the bone age according to Greulich-Pyle standards [11], evaluated every 12 months. Index of bone age delay was calculated from the bone age to chronological age ratio (BA/CA) before, during and at the end of rhGH treatment.

\section{Statistical analysis}

A database was prepared in the calculative sheet Excel v. 2007. Detection of outlying values was carried out using the criterion of $\operatorname{abs}(\mathrm{X})>\mathrm{Q}+1.5 \mathrm{IQR}$; IQR indicating interquartile range, $\mathrm{Q}$-upper or lower quartile of distribution of the random variable X. Normality of distribution was checked using parametric Lilliefors test. Hypothesis of homogeneity of the variance was verified by Bartlett statistics. Comparative analysis of the groups for variables of normal distribution was carried out using standard ANOVA algorithm and the Student's $t$ test. In the case of non-Gaussian distribution and/or lack of homogeneity of the variance, hypothesis equality of distributions among subgroups was verified using rank non-parametric method of ANOVA Kruskal-Wallis and the Mann-Whitney $U$ test. Comparative analysis of observed time changes was carried out using Friedmann ANOVA test as well as the Wilcoxon signed-rank test or the Student's $t$ test. Results of the analyses were considered significant when $p$ level was below 0.05 .

Patients' guardians and patients older than 16 years of age signed informed consent for the diagnostic procedures as well as for the rhGH treatment. The study was approved by the Ethics Committee of the Medical University of Silesia.

\section{Results}

The initial analysis showed no significant differences between genders in the aspect of assessed parameters, thus girls and boys were further evaluated as one group.

On the basis of MRI, three subgroups were designated: (1) children with normal H-P area, NP (63 children, $74.1 \%$ ); (2) empty sella syndrome, (ESS) (12 children, 14.1\%; Fig. 1a, b); (3) ectopic posterior pituitary (EPP) (ten children, 11.8\%; Fig. 2a, b). When the interrupted or poorly visualised pituitary stalk, pituitary hypoplasia and ectopy of the pituitary co-existed, the latter determined affinity to the EPP group. Structural abnormalities in the pituitary MRI in total were found in $22(25.9 \%)$ children. Additionally, hypoplasia of the corpus callosum along with septum pellucidum agenesis were visualised in one girl, and Arnold Chiari I malformation was found in one boy.

Children in the ESS group were significantly younger (median, 10.9 years; range, 6.9-15.4 years) in comparison to NP children (median, 14.2 years; range, 7.7-18.7; $p<$ 0.001 ) and the EPP group (median, 14.9 years; range, 7.017.2 years; $p=0.045$ ), whereas the chronological age of two latter groups was comparable $(p=0.606)$. Most children with structural abnormalities in the H-P area were either prepubertal or in an early stage of puberty, whereas NP children had already entered puberty.

Combined pituitary hormonal deficiency was diagnosed in nine children: in five cases of EPP, three ESS and in one case of NP.

Relations between auxologic parameters and structural abnormalities of the hypothalamic-pituitary area before rhGH therapy

Children with EPP were significantly shorter as compared to children in the NP group (median hSDS, -3.8 vs -3.1 , respectively; $p=0.049$ ), whereas height between EPP and
Fig. 1 MRI of the partially empty sella (circle), SE/T1, coronary plane (a) and sagittal plane (b)
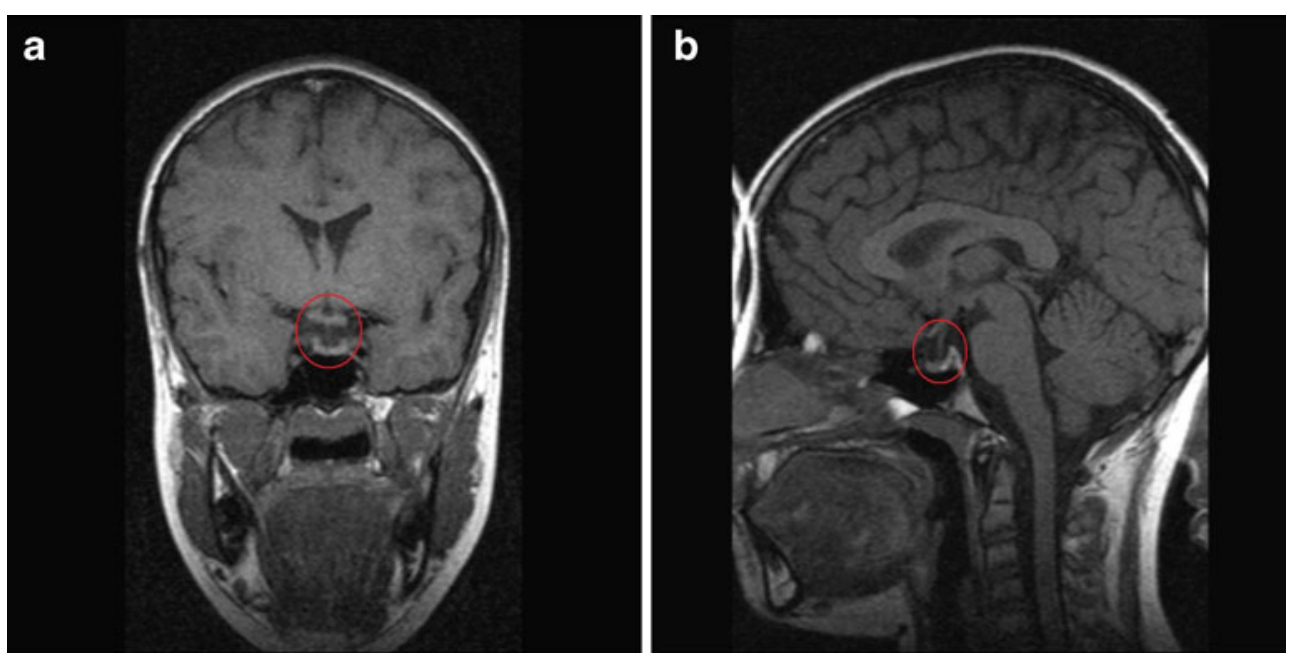
Fig. 2 MRI of the ectopic posterior pituitary (arrow), SE/T1, coronary plane (a) and sagittal plane (b)
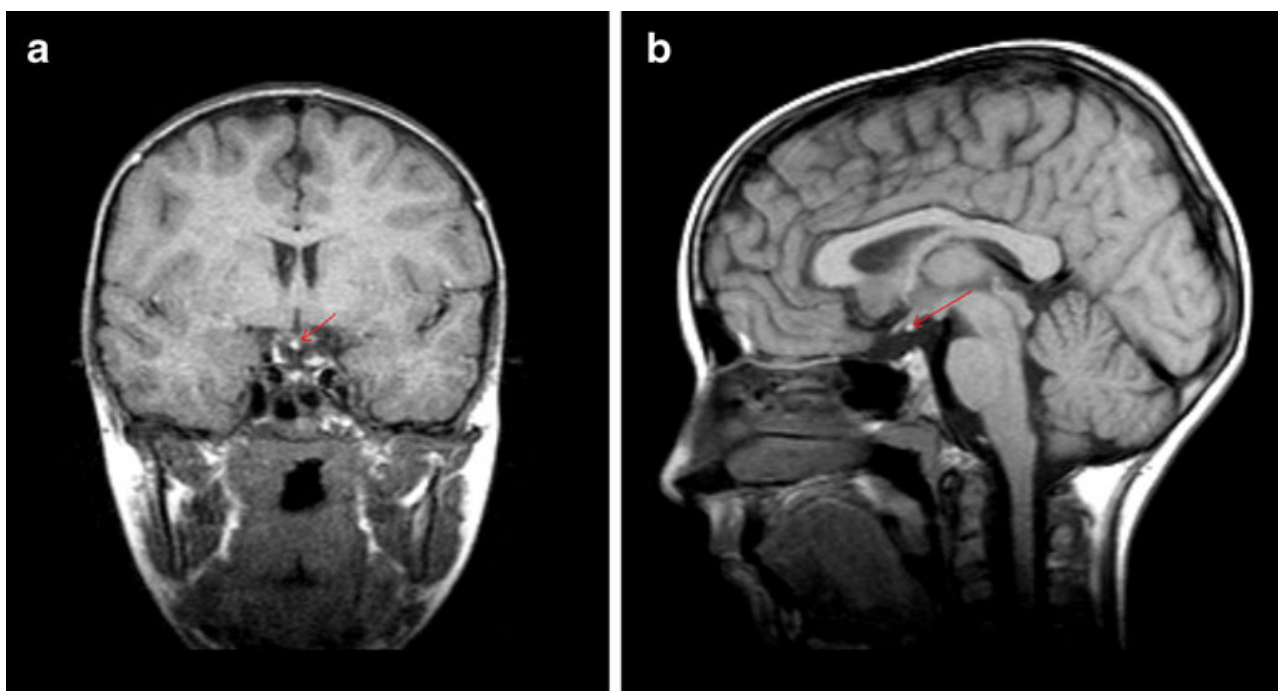

ESS, as well as between ESS and NP did not differ significantly.

Moreover, patients with EPP were characterized by the greatest deviation from the mid-parental height, as compared to two other groups. Median hSDS-mpSDS for the EPP amounted to -2.5 vs -1.5 for ESS and -1.7 for NP. Statistical differences were significant between EPP and NP $(p=0.028)$. After excluding outlying values, the ESS group was characterized by the greatest dispersion of the examined variable.

In contrast, bone delay index (BA/CA) was the lowest among children with ESS, as compared to NP and EPP (median BA/CA was equal to $0.69 ; 0.82 ; 0.77$, respectively); however, the statistical significance was found only between NP and ESS $(p=0.037)$

Relations between auxologic parameters and structural abnormalities of the hypothalamic-pituitary area after the first year of rhGH therapy

Analysis of patients' height and their deviation from the mid-parental height after the first year of rhGH therapy did not show significant differences among examined groups. In contrast, differences were observed while comparing bone delay index, which was significantly lower in the EPP and ESS (median BA/CA, 0.81; 0.74, respectively) as compared to NP $(0.87 ; p=0.042$ and 0.001 , respectively).

Relations between auxologic parameters and structural abnormalities of the hypothalamic-pituitary area after termination of rhGH therapy

After discontinuation of $\mathrm{rhGH}$ therapy, the bone age remained the most delayed in the EPP group (BA/CA, $0.82)$, as compared to NP $(0.89 ; p=0.006)$ and $\operatorname{ESS}(0.91$; $p=0.029)$. Other auxologic parameters were comparable in all groups.

Height velocity in relation to the pituitary MRI prior, during and after GH therapy

Height velocity, expressed by a change of hSDS between two time intervals (delta hSDS) did not differ significantly among the groups before rhGH treatment. Children with EPP showed a tendency to slower height velocity as compared with the two remaining groups; however, without statistical significance.

In contrast, during rhGH therapy the EPP group turned to the greatest positive change of hSDS in comparison to the NP and EPP groups (Table 1; Fig. 3). In the following years, change of hSDS was comparable in all the groups.

\section{Discussion}

According to various reports, patients with GHD and structural abnormalities in MRI of the H-P area differ from subjects with normal image of the pituitary in terms of auxologic parameters $[6,18,24,26]$. Coutant et al. showed that children with GHD and abnormal MRI of the H-P area were shorter and younger at the referral [6]. Additionally, Maghnie et al. found the bone age to be more delayed in this group of patients [18]. Similarly, we observed differences in the studied groups, namely children with EPP were shorter, whereas the ESS group manifested more delayed bone age as compared to subjects with normal pituitary. Moreover, the height of EPP patients showed the greatest deviation from the mid-parental height as compared to the two other groups. The latter finding may indicate that growth in children with normal pituitary, as well as in 
Table 1 Height velocity (delta hSDS) in the first 12 months of rGH treatment depending on the MRI of the H-P region

\begin{tabular}{lllll}
\hline Delta hSDS & Median & Range & Mean & SD \\
\hline NP $(n=63)$ & 0.6 & $-1.1 ; 1.8$ & 0.7 & 0.6 \\
ESS $(n=12)$ & 0.5 & $-0.4 ; 1.6$ & 0.7 & 0.6 \\
EPP $(n=10)$ & 0.9 & $-0.1 ; 2.1$ & 1.0 & 0.8 \\
\hline
\end{tabular}

Significance levels for differences among the groups: 1 vs $2 p=0.578$; 1 vs $3 p=0.042 ; 2$ vs $3 p=0.040$

$N P$ normal pituitary, ESS empty sella syndrome, EPP ectopic posterior pituitary

children with features of empty sella can be significantly influenced by familial components. Studies in the adult population indicate that most cases of ESS are asymptomatic [7]. In contrast, children more often manifest hormonal and growth disturbances [30]. Hence, it has been disputed if radiological features of partially empty sella translate into hormonal dysfunction of the H-P axis, or it is only a temporary phenomenon and the small pituitary normalizes its parameters in the course of time [18].

Analyzing bone age, we found that it advanced in the slowest manner in the EPP group, indirectly prolonging the growth process. Not until the sex hormone deficiency becomes overt and yet not compensated, maturation of the bone age may be relatively slow. In our study, combined pituitary hormonal deficiency co-existed with structural midline defects in eight out of nine cases. It may be hypothesized that in the case of EPP, hormonal deficiencies evolve gradually, depending on the degree of the connection between the hypothalamus and the pituitary. However, Tauber et al. found that children with severe pituitary hypoplasia and ectopic posterior lobe may present with symptoms of GH deficiency as early as in the neonatal

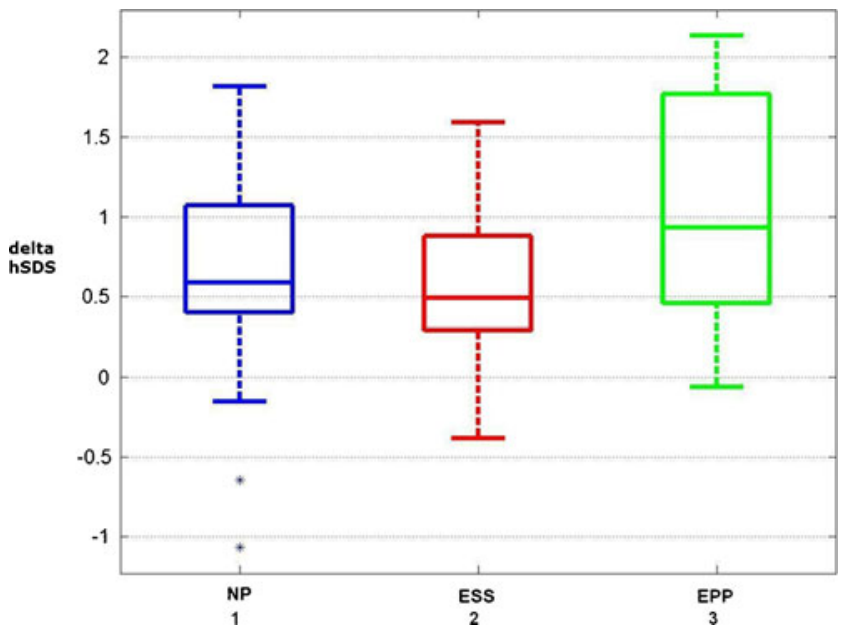

Fig. 3 Box plots presenting height velocity (delta hSDS) in the first 12 months of rGH treatment depending on the MRI of the H-P region. $N P$ normal pituitary, ESS empty sella syndrome, EPP ectopic posterior pituitary. Asterisks represent outlying values period [24]. The degree of pituitary dysfunction may depend on the localisation of the posterior lobe, namely whether it is placed in the median eminence or along the stalk [5]. Status of the vascular bed which takes part in signalisation between the hypothalamus and the pituitary is also relevant. Precise dynamic MRI examination using contrast agents facilitates visualisation of potential pathology in this domain $[4,19]$. Unfortunately in our study, it was not possible to discriminate the subgroups in terms of exact localisation of the posterior lobe due to a small number of subjects.

Height velocity may be considered as a marker of the response to rhGH. Change of hSDS did not differ significantly among the studied groups before the treatment, which is justified by the fact that slow growth rate was one of the inclusion criteria, common for all subjects. Subsequently, height velocity improved in all patients during the observational period. Most children achieved height, which did not differ significantly from the familial potential, fulfilling therapeutic goals in the whole cohort. However, children with ectopy of the posterior pituitary showed the greatest positive change of the hSDS value, as observed in the first year of the treatment. It reflects the most dynamic catch-up growth in this group of patients, starting from the greatest height deficit in the reference to peers and parents. Literature reports show strong correlation between the height velocity in the first year of rhGH treatment, particularly in the prepubertal period, and the total increment of hSDS [20, 22]. Moreover, Zenaty et al. showed that the presence of congenital abnormalities in the H-P area on MRI was more important than the maximum concentration GH to predict the growth response to rhGH treatment in prepubertal GH-deficient children during a 3-year observation [29]. In our study, most patients with abnormal H-P image were prepubertal at the referral, in contrast to children with normal pituitary. In connection with the above, the former group would have the most rational chance to optimize the final height.

Interesting findings come from Coutant et al. who did not show any significant differences in height velocity between children with idiopathic short stature and GHdeficient patients, both groups treated with $\mathrm{rhGH}$, providing normal MRI of the pituitary was found [6]. Basing on these findings, the authors hypothesised that short stature in the latter group, at least in part, is conditioned by extrahormonal factors. It was observed that even patients with severe GHD showed similar height velocity to the group with so-called partial GHD, if the MRI of the pituitary was normal. The more rapid height velocity was observed in severe GHD, co-existing with structural abnormalities [6]. There are doubts if non-treated children with normal pituitary image would be able to grow spontaneously, for example at the time of puberty. Difficulties in answering 
this question unequivocally result from poor ethical possibility to select a control group comprising children with laboratory indicators of GHD, not submitted to rhGH therapy.

It must be underlined, however, that not only midline defects condition GH deficiency. Mutations POU1F1 and PROP1, which result in combined pituitary hormonal deficiency, present with various sizes of the pituitary, from hypertrophy to aplasia [28]. In the case of GH1 mutations, despite severe GH deficiency, the structure of the pituitary MRI is usually normal, as there is functional but not structural impairment of somatotropes [2]. Thus, it may be assumed that MRI of the pituitary depends on the stage of life when it is performed $[16,18]$.

Basing on the results of this study and assuming critical point of view, it cannot be stated explicitly that structural abnormalities of the H-P region conditions the final height. However, the greatest catch-up growth in the essential first year of rhGH therapy in the group with ectopic posterior pituitary indicates the best responders and may even influence titration of the rhGH dosage. However, this hypothesis would demand further randomized studies.

Nevertheless, MRI constitutes an essential method in diagnosing endocrine disturbances, including cases of congenital aetiology. It also provides additional information concerning response to the substitution therapy. Dynamically improving MRI methods with tridimensional acquisitions, determining the real size of the gland, and dynamic studies with contrast agents may contribute to better understanding the function of the hypothalamic-pituitary axis.

Open Access This article is distributed under the terms of the Creative Commons Attribution Noncommercial License which permits any noncommercial use, distribution, and reproduction in any medium, provided the original author(s) and source are credited.

\section{References}

1. Argente J, Abusrewil SA, Bona G, Chiarelli F, Kelnar CJ, Skordis N (2001) International workshop on management of puberty for optimum auxological results isolated growth hormone deficiency in children and adolescents. J Pediatr Endocrinol Metab 14(suppl 2):1003-1008

2. Binder G, Nagel BHP, Ranke MB, Mullis PE (2002) Isolated GH deficiency (IGHD) type II: imaging of the pituitary gland by magnetic resonance reveals characteristic differences in comparison with severe IGHD of unknown origin. Eur J Endocrinol 147:755-760

3. Bozzola M, Adamsbaum C, Biscaldi I, Zecca M, Cisternino M, Genovese E, Richard I, Kalifa G, Chaussain JL (1996) Role of magnetic resonance imaging in the diagnosis and prognosis of growth hormone deficiency. Clin Endocrinol (Oxf) 45:21-6
4. Castillo M (2005) Pituitary gland: development, normal appearances and Magnetic Resonance imaging protocols. Top Magn Reson Imaging 4:259-268

5. Chen S, Leger J, Garel C, Hassan M, Czernichow P (1999) Growth hormone deficiency with ectopic neurohypophysis: anatomical variations and relationship between the visibility of the pituitary stalk asserted by magnetic resonance imagining and anterior pituitary function. J Clin Endocrinol Metab 84:24082413

6. Coutant R, Rouleau S, Despert F, Magontier N, Loisel D, Limal J-M (2001) Growth and adult height in GH-treated children with nonacquired GH deficiency and idiopathic short stature: the influence of pituitary magnetic resonance imaging findings. J Clin Endocrinol Metab 86:4649-4654

7. de Marinis L, Bonadonna S, Bianchi A, Maira G, Giustina A (2005) Primary empty sella. J Clin Endocrinol Metab 91:33293336

8. Elster AD (1993) Modern imaging of the pituitary. Radiology 187:1-14

9. Garel G, Leger J (2007) Contribution of magnetic resonance imaging in non-tumoral hypopituitarism in children. Horm Res 67:194-202

10. Ghigo E, Bellone J, Aimaretti G, Bellone S, Loche S, Cappa M, Bartolotta E, Dammacco F, Camanni F (1996) Reliability of provocative tests to assess growth hormone secretory status. Study in 472 normally growing children. J Clin Endocrinol Metab 81:3323-3327

11. Greulich WW, Pyle SI (eds) (1959) Radiographic atlas of skeletal development of the hand and wrist, 2nd edn. Stanford University Press, Stanford

12. Guyda HJ (1999) Four decades of growth hormone therapy for short children. What have we achieved? J Clin Endocrinol Metab 84:4307-4316

13. Guyda HJ (2000) Growth hormone testing and the short child. Pediatr Res 48:579-580

14. Hilczer M, Smyczyńska J, Lewinski A (2006) Limitations of clinical utility of growth hormone stimulating tests in diagnosing children with short stature. Endocr Regul 40:69-75

15. Kalina M, Kalina-Faska B, Gruszczyńska K, JanuszekTrzciąkowska A, Kluczewska E, Małecka-Tendera E (2007) Relationship between magentic resonance image of the pituitary gland and growth hormone concentrations in short-stature children with growth hormone deficiency. Endokrynol Pediatr 6:35-44

16. Leger J, Danner S, Simon D, Garel C, Czernichow P (2005) Do all patients with childhood-onset growth hormone deficiency (GHD) and ectopic neurohypohysis have persistent GHD in adulthood? J Clin Endocrinol Metab 90:650-656

17. Maghnie M, Triulzi F, Larizza D, Preti P, Priora C, Scotti G, Severi F (1991) Hypothalamic-pituitary dysfunction in growth hormone-deficient patients with pituitary abnormalities. J Clin Endocrinol Metab 73:79-83

18. Maghnie M, Strigazzi C, Tinelli C, Autelli M, Cisternino M, Loche S, Severi F (1999) Growth Hormone (GH) deficiency (GHD) of childhood onset: reassessment of GH status and evaluation of the predictive criteria for permanent GHD in young adults. J Clin Endocrinol Metab 84:1324-1328

19. Maghnie M, Salati B, Bianchi S, Rallo M, Tinelli C, Autelli M, Aimaretti G, Ghigo E (2001) Relationship between the morphological evaluation of the pituitary and the growth hormone (GH) response to GH-releasing hormone plus arginine in children and adults with congenital hypopituitarism. J Clin Endocrinol Metab 86:1574-1579

20. Maghnie M, Ambrosini L, Cappa M, Pozzobon G, Ghizzoni L, Ubertini MG, di Iorgi N, Tinelli C, Pilia S, Chiumello G, Lorini R, Loche S (2006) Adult height in patients with permanent growth 
hormone deficiency with and without multiple pituitary hormone deficiencies. J Clin Endocrinol Metab 91:2900-2950

21. Palczewska J, Niedźwiecka Z (2001) Wskaźniki rozwoju somatycznego dzieci i młodzieży warszawskiej. Med Wieku Rozw 2 (suppl 1):55-56, 113-114

22. Reiter EO, Price DA, Wilton P, Albertsson-Wikland K, Ranke MB (2006) Effect of growth hormone (GH) treatment on the near-final height of 1258 patients with idiopathic GH deficiency: analysis of a large international database. J Clin Endocrinol Metab 91:2047-2054

23. Rosenfeld RG, Albertsson-Wikland K, Cassorla F, Frasier SD, Hasegawa Y, Hintz RL, Lafranchi S, Lippe B, Loriaux L, Melmed S (1995) Diagnostic controversy: the diagnosis of childhood growth hormone deficiency revisited. J Clin Endocrinol Metab 80:1532-1540

24. Tauber M, Chevrel J, Diene G, Moulin P, Jouret B, Oliver I, Pienkowski C, Sevely A (2005) Long-term evolution of endocrine disorders and effect of GH therapy in 35 patients with pituitary stalk interruption syndrome. Horm Res 64:266-273

25. Tillmann V, Tang VW, Price DA, Hughes DG, Wright NB, Clayton PE (2000) Magnetic resonance imaging of the hypothalamic-pituitary axis in the diagnosis of growth hormone deficiency. J Pediatr Endocrinol Metabol 13:1577-1583
26. Triulzi F, Scotti G, di Natale B, Pellini C, Lukezic M, Scognamiglio M, Chiumello G (1994) Evidence of a congenital midline brain anomaly in pituitary dwarfs: a magnetic resonance imaging study in 101 patients. Pediatrics 93:409-416

27. Van der Broeck J, Hering P, Van de Lely A, Hokken-Koelega A (1999) Interpretative difficulties with growth hormone provocative retesting in childhood-onset growth hormone deficiency. Horm Res 51:1-9

28. Voutetakis A, Sertedaki A, Livadas S, Xekouki P, Bossis I, DacouVoutetakis C, Argyropoulou MI (2006) Pituitary size fluctuation in long-term MR studies of PROP1 deficient patients: a persistent pathophysiological mechanism? J Endocrinol Invest 29:462-466

29. Zenaty D, Garel C, Limoni C, Czernichow P, Leger J (2003) Presence of magnetic resonance imagining abnormalities of the hypothalamic-pituitary axis is a significant determinant of the first 3 years growth response to human growth hormone treatment in prepubertal children with nonaquired growth hormone deficiency. Clin Endocrinol (Oxf) 58:647-652

30. Zucchini S, Ambrosetto P, Carla G, Tani G, Franzoni E, Cacciari E (1995) Primary empty sella: differences and similarities between children and adults. Acta Paediatr 84:1382-1385 